\title{
Conceptions de l'innovation et formations de l'ingénieur
}

\author{
Marianne Chouteau, Joëlle Forest, Céline Nguyen \\ Université de Lyon, EVS-ITUS UMR 5600, INSA de Lyon
}

\section{Introduction}

Ceux que l'on identifie aujourd'hui comme ayant été les premiers ingénieurs pendant l'Antiquité étaient le plus souvent spécialisés dans la construction des machines de guerre et des navires. Bruno Latour (Latour, 2006) évoque comment le roi Hiéron, impressionné par la puissance des outils techniques conçus par Archimède, lui demanda de lui construire une machine de guerre dédiée à la défense de Syracuse. Nullement formé dans une école, Archimède est pourtant considéré comme l'un des premiers ingénieurs, utilisant la ruse et l'ingéniosité pour combattre la force.

En France, on situe la préhistoire des écoles d'ingénieurs au XVIIe siècle. Jusqu'en 1763/64 les jésuites géraient environ 120 collèges pour former, entre autres, du personnel technique. A la fin du XVIIe siècle, Vauban instaure cependant les premières classes préparatoires, inspirées du modèle des collèges jésuites. Les premières écoles d'Etat commencent alors à apparaître : l'Ecole Royale des Ponts et Chaussées en 1747 suivie, en 1783, par l'Ecole Royale des Mines ${ }^{1}$. L'Ecole Royale des Ponts et Chaussées répondait à un besoin social important en infrastructures. En effet, sur le territoire français, les échanges se développent et de ce fait, les besoins en ponts, routes et chemins de fer se font plus pressants. En 1794, l'Ecole centrale des travaux publics voit le jour et deviendra l'Ecole polytechnique en 1795. Les objectifs pédagogiques affichés par ces écoles d'Etat visent à former du personnel technique de haut niveau. Les élèves admis dans ces écoles d'État reçoivent alors une éducation tant scientifique que militaire.

Pendant un siècle, la formation d'ingénieurs sera un monopole d'Etat. Les ingénieurs sont des fonctionnaires civils ou militaires. La formation est axée sur des connaissances scientifiques pointues (mathématiques, physique) privilégiant un raisonnement déductif et complétées par une formation sociale aristocratique (danse, équitation, escrime, histoire de l'art, gymnastique). Les élèves intègrent des classes préparatoires où la sélection est très importante.

Jusqu'en 1837 un ingénieur est considéré comme «un ancien élève de l'Ecole polytechnique, il fera carrière dans l'un des Corps de l'Etat ; le Génie militaire, l'Artillerie, les Mines et les Ponts et chaussées sont cités comme les plus importants - où il aura, grâce à son intelligence et à ses exceptionnelles qualités de caractère, une fonction de premier plan dans le domaine de la défense nationale aussi bien que dans tous les autres rouages de l'Etat $»^{2}$. Toutefois, ces ingénieurs de haut rang accèdent à des postes à responsabilité non pas pour y exercer leurs compétences scientifiques ou techniques mais pour y exercer des fonctions administratives et décisionnaires.

Les besoins de l'industrie devenant plus importants se crée alors un groupe professionnel non fonctionnaire distinct des ingénieurs d'Etat. Ce groupe professionnel sera longtemps représenté par les gadzarts des Arts et métiers (Shinn, 1978 : 49) mais pas seulement. En

\footnotetext{
${ }^{1}$ Ces écoles existent toujours aujourd'hui. L'Ecole Royale des Ponts et Chaussées est devenue l'Ecole des chaussées Paris-Tech.

${ }^{2}$ Ce propos de C. Martin date de 1837 et paraît dans Vocabulaire de la langue française. Il est cité par Terry Shinn (1978).
} 
1828, 1'Ecole Centrale de Paris $^{3}$ ouvre ses portes pour former des ingénieurs civils (Vérin, 1993 : 36).

Cette distinction entre types d'ingénieurs se perpétuera approximativement jusqu'à la veille de la première guerre mondiale où s'opère un premier changement. L'ingénieur reste un scientifique mais son savoir doit servir à la transformation des matériaux (Shinn, 1978). Ce n'est pas un savoir scientifique «théorique » mais un savoir appliqué qui est requis et dispensé dans les écoles d'ingénieurs.

Un second changement a lieu après la seconde Guerre mondiale. La France a besoin de renouveau tant au niveau économique que scientifique, technique ou social. Après la seconde guerre mondiale et avec l'engagement progressif dans la Guerre froide, la reconstruction de la France et le développement de nouvelles industries s'appuyant sur des savoirs scientifiques et techniques requièrent des ingénieurs en nombre. C'est au cours des décennies entre 1950 et 1980 que naîtront les Instituts Nationaux des Sciences Appliquées (INSA) ou les Ecoles Nationales d'Ingénieurs (ENI) (Grelon, 1993).

Si l'histoire des ingénieurs que nous avons esquissée précédemment permet de comprendre que la figure de l'ingénieur a évolué au fil du temps (de l'ingénieur d'Etat à l'ingénieur spécialisé et engagé dans l'industrie), elle permet aussi de comprendre comment la figure de l'ingénieur conduit à infléchir la façon dont est pensée sa formation. Dans la présente contribution nous souhaitons pousser plus en avant la question de la formation des ingénieurs en expliquant comment la conception même de la technique émaille la formation des ingénieurs.

Nous montrerons comment la prise en compte du rôle clé de la conception dans l'innovation ${ }^{4}$ s'est substituée à la vision de l'innovation comme application des sciences et comment ce changement de paradigme conduit à repenser le modèle de formation des ingénieurs et la place des sciences humaines et sociales au sein de la dite formation.

\section{Emergence d'une pensée technique}

Le concept d'innovation émerge à partir du moment où la technique n'est plus pensée comme reproduction du monde, mais comme conception du monde. En effet, l'histoire de la technique montre que dans les sociétés archaïques les objets techniques sont vus comme une imitation de la nature (Chabot, 2003 : 22). La pensée grecque n'est pas «technologique » au sens contemporain du terme. Si elle laïcise la technique (la sortant de la magie et des interventions divines invoquées jusque-là pour expliquer les prouesses techniques), elle est un âge de «stagnation technique », sans véritable "pensée technique » malgré la qualité de son " outillage intellectuel » (Lamard et Lequin, 2006 : 28). Comme le souligne François Sigaut «Lorsqu'elles sont sans mystère, elles appartiennent à la routine où à la servilité, et ne valent pas la peine qu'on en parle. Lorsqu'au contraire elles surprennent par leur nouveauté, par l'habileté qu'elles impliquent ou par leur caractère inhabituel, antinaturel de leurs effets, elles deviennent ruses ou artifices (...) mais en aucun cas les techniques ne sont un produit légitime de la droite raison ». (Sigaut, 1986 : 17). La connotation négative dont sont chargées les techniques dans la conception aristotélicienne les laissent en dehors de la boucle de la pensée (Simondon, 2001 ; Stiegler, 2004).

La Renaissance européenne consacre l'innovation comme vecteur d'émancipation et de transformation du monde et marque un intérêt nouveau pour la culture technique. L'Encyclopédie de Diderot et d'Alembert (1751-1772) est un premier pas en ce sens : "Les arts mécaniques dépendant d'une opération manuelle, et asservis, qu'on me permette ce

\footnotetext{
${ }^{3}$ L'Ecole Centrale de Lyon ouvrit quant à elle ses portes en 1857 sous l'impulsion d'industriels lyonnais dont François Arlès-Dufour et Désiré Girardon

${ }^{4}$ Dans cette contribution, notre discours portera exclusivement sur l'innovation technique.
} 
terme, à une espèce de routine, ont été abandonnés à ceux d'entre les hommes que les préjugés ont placés dans la classe la plus inférieure [...] La découverte de la boussole n'est pas moins avantageuse au genre humain, que ne le serait à la physique l'explication des propriétés de cette aiguille. [...] Cependant, c'est peut-être chez les artisans qu'il faut aller chercher les preuves les plus admirables de la sagacité de l'esprit, de sa patience et de ses ressources » (D'Alembert, $2010: 47-48$ ).

Le projet des Encyclopédistes est de rassembler le connu en vue de le diffuser et ouvre ainsi la voie à une technologie descriptive. Si la préoccupation est classificatoire et si elle tient de la volonté de démocratiser la technique, elle ouvre aussi l'espace à l'élaboration du concept de technologie, inventé par Beckmann en 1770. C'est cependant Jacob Bigelow, qui systématise l'usage du mot technologie dans son ouvrage Elements of technology (1829) tout en le dépouillant de la dimension philosophique et politique qu'il avait chez Beckmann. Jacob Bigelow défend en effet la vision d'une science tout entière mobilisée par ses applications techniques. Cette conception de la technologie entendue comme une pratique efficace fondée sur l'application des sciences a permis au modèle linéaire et hiérarchique, ou modèle de la big science, de s'instituer comme le modèle d'innovation dominant qui aida dès le début des années 1950 à définir les orientations des premières politiques de la recherche et de l'innovation.

La conception de Jacob Bigelow a également fourni un modèle de formation des ingénieurs illustré par le Massachusetts Institute of Technology (MIT) qui, au niveau français a notamment été évoqué lors de la création de l'INSA en 1957. Henri Longchambon, l'un des fondateurs de l'institut écrit en effet dans un article paru dans la Revue de l'enseignement supérieur en 1957 que l'école d'ingénieur américaine est une source d'inspiration: "si trop tenus par nos traditions, nous étions incapables d'en imaginer les bases, nous pourrions puiser ailleurs, dans d'autres pays, des exemples ayant fait leurs preuves, notamment auprès du Massachusetts Institute of Technology, dont personne ne contestera qu'il constitue une des meilleures "écoles d'ingénieurs du monde» (Longchambon, 1957: 13). Le modèle de formation inspiré de Jacob Bigelow n'est pas neutre car il va diffuser le modèle de l'ingénieur comme « problem solver ».

\section{Vers un modèle de formation alternatif ?}

Dans les années 1960, la vision prédominante de l'activité de l'ingénieur est en effet clairement celle de l'ingénieur qui résoud des problèmes : "engineering is the profession in which a knowledge of the mathematical and natural sciences gained by study, experience, and practice is applied with judgement to develop ways to utilize, economically, the materials and forces of nature for the benefit of mankind» (Engineers' Council for Professional development, 1968) et non celle de l'ingénieur concepteur ou ingénieux, ce que révèle le point de vue de l'ASEE (American Society for Engineering Education) : "The ASEE Goals Report (ASEE, 1968) states that the engineer of the future-like the engineer of the past- must become a skillful problem solver»(Waina, 1969). La conception des enseignements de l'époque est alors fondée sur l'apprentissage des connaissances scientifiques de base permettant de résoudre tel ou tel problème.

Comme l'a souligné Herbert Simon dès 1969 au début de son chapitre consacré à la science de la conception "par un paradoxe ironique, alors que s'affirme le rôle décisif de la conception dans toute activité professionnelle, les sciences naturelles, surtout dans les deux ou trois décennies qui ont suivi la seconde guerre mondiale, ont presque complètement éliminé les sciences de l'artificiel du programme des écoles formant des professionnels, les écoles d'ingénieurs sont devenues des écoles de physique et de mathématique... » et de 
poursuivre "l'usage du qualificatif "appliqué" dissimule le fait, mais ne le change pas» (Simon, 1969).

Ce constat a été actualisé quelques années plus tard par le National Research Council soulignant que «les programmes de cycle d'ingénieur dédiés aux nouvelles théories et méthodologies de conception sont en nombre vraiment insuffisants pour produire les diplômés qualifiés dans le domaine dont ont besoin les entreprises industrielle» (National Research Council, 1991 : 44).

L'importance de l'adéquation de la formation des ingénieurs à leur future activité n'est pas une question triviale. C'est elle qui a conduit le Journal of Engineering Education à revoir ses missions en 2003 (Felder \& alii, 2005). C'est également elle qui a incité en 2006, le Special Report du Journal of Engineering Education intitule The research agenda for the new discipline of engineering education à lancer un appel à la nation «our nation needs to make the critical research investments that will transform today's educational system into the preeminent paradigm for engineering education and ensure that the U.S. maintains its leadership role in adressing the global challenges of the future »(JEE, $2006: 261)$.

Cette relative occultation de la conception dans les programmes d'enseignement est dommageable car les écoles d'ingénieurs ont de toute évidence privilégié le modèle de la raison platonicienne, analytique, académique, délaissant celui de la raison ingénieuse (Faucheux, Forest, 2011). Or, l'enjeu pour les écoles d'ingénieurs n'est-il pas précisément de privilégier cette pensée, de la démarquer de la pensée opératoire, appliquée, qui la caricature souvent ou en tous les cas la masque?

En effet, et comme l'avait déjà souligné Gaston Berger évoquant la capacité de l'homme à créer des objets techniques, notre rapport à la technique n'est pas celui d'une simple application des sciences puisque l'inventeur ne peut se contenter d'appliquer et de reproduire : "L'invention a partout les mêmes traits généraux; ici ou là, elle exige toujours un esprit ouvert et attentif, l'art des rapprochements inattendus, quelques hasards heureux que l'on sait saisir et utiliser, l'aptitude à se concentrer, la ténacité dans le travail, la rigueur dans la critique (Berger, $1958: 6$ ).

Les écoles d'ingénieurs apparaissent ainsi comme le lieu où doit être pensée l'invention : «Le mot "technique" revient dans tous les programmes; il est exalté dans tous les discours officiels. Pourtant, beaucoup de nos contemporains ne voient pas clairement le caractère universel de la technique et se posent d'illusoires problèmes»(Berger, 1958: 5), et ce d'autant plus que, dans l'esprit de Gaston Berger penser l'invention technique, c'est s'extraire de la conception de l'innovation comme "simple » application des sciences au profit d'une conception de la technique qui nous donne les moyens de nous penser nous-mêmes. La technique est, par les créations qu'elle rend possibles, un moyen privilégié et peu reconnu de penser notre action sur le monde, de penser notre rapport au monde voire même de penser notre humanité. Cette vision nous invite alors à penser la nécessité d'inclure de manière centrale les sciences humaines et sociales (SHS) dans la formation au métier d'ingénieur « concepteur».

\section{Quelle place des SHS dans la formation de l'ingénieur?}

Denis Lemaître (2007), considérant les formations en Humanité au sein des écoles d'ingénieurs, a proposé une typologie correspondant à trois modèles de formation.

Le premier s'inscrit dans l'idéal humaniste des Lumières et vise à élever l'âme des futurs ingénieurs: "le point de vue idéaliste qui inspire ce type de formation suppose que l'acquisition d'une culture générale, la maîtrise des savoirs les plus élevés, le partage d'un héritage savant, sont le préalable à une action juste et efficace au service du progrès" (Lemaître, 2007 : 64). Le second, plus récent, incarne les savoirs dans l'action et sont tournés vers des savoirs appliqués, notamment au sein du monde de l'entreprise : management de 
projet par exemple. Enfin, le troisième vise à délivrer des connaissances dont l'application servira au développement personnel des étudiants ; l'auteur cite notamment les enseignements de communication.

Si Denis Lemaître souligne que «Ces différents modèles existent rarement à l'état pur dans une institution de formation, mais se retrouvent comme archétypes dans des configurations à chaque fois différentes. » (Sonntag et al. 2008), il constate pour autant une évolution d'une vision idéaliste de la connaissance à une vision plus pragmatique, les enseignements de sciences humaines et sociales visant de plus en plus l'adaptation des individus aux activités professionnelles et aux réalités économiques : "L'objectif est de transmettre des savoirs opérationnels qui conduisent à une certaine spécialisation des individus » (Sonntag et al. 2008). De fait, on sort là du modèle instauré par les Grandes Ecoles créées à l'époque des Lumières qui voyaient dans l'ingénieur celui par qui devait arriver le progrès : "Les valeurs universalistes des humanités classiques sont remplacées par des valeurs de l'efficacité pratique, de l'adaptation aux activités humaines et aux conditions sociales observables. » (Sonntag et al. 2008).

Comme le souligne Dominique Vinck si la qualité des enseignements en sciences humaines et sociales ainsi que leur utilité pour la formation de l'individu ne sont pas à remettre en cause, en revanche, "leur contribution au développement d'une capacité réflexive portant sur l'action technique et industrielle est insignifiante » (Vinck, 2007).

Est ainsi posée la question de la place et de la contribution des SHS à la formation des ingénieurs. Comment les SHS peuvent-elles permettre de dépasser le modèle de l'innovation comme application des sciences et, en même temps, comment ce dépassement impacte-t-il le fonctionnement des SHS en écoles d'ingénieur? N'est-ce pas un moyen, pour reprendre la typologie de Denis Lemaitre, de développer une quatrième voie qui requiert le rapprochement entre SHS et SPI (sciences pour l'ingénieur) mais aussi entre les SHS elles-mêmes ? Former à l'innovation, c'est en effet former à la réalisation technique mais c'est aussi penser la société dans laquelle cette réalisation prend place. Le développement d'une innovation ne consiste pas seulement à trouver la "meilleure » solution technologique encore faut-il qu'elle soit en phase avec les attentes des différentes parties prenantes (usagers, collectivités, fabricants, etc.). Cette quatrième voie implique donc de ne pas dissocier la technique de la société et de l'humain. Par là même, elle oblige à adopter une vision qui dépasse les frontières disciplinaires habituelles et à envisager, à terme, un bouleversement de la cartographie traditionnelle des savoirs. »

Du point de vue de la pédagogie, une formation à l'innovation ne peut faire l'économie d'une approche transversale qui permet de convoquer une analyse plutôt sociologique des acteurs engagés dans l'innovation (types d'acteurs et de relations ${ }^{5}$ ) et des points de vue historique, politique et économique : concurrence entre innovations, médiation du politique en faveur d'une innovation, intervention d'un cadre législatif, etc.

Le futur ingénieur ne peut en effet ignorer que les innovations qu'il concevra nécessitent de comprendre la complexité des agencements entre le social et la technique et de se départir d'un déterminisme social ou technique. C'est une façon de comprendre comment les innovations apparaissent puis disparaissent et quels bouleversements sociaux elles engendrent mais aussi un moyen de se dégager du stéréotype de l'invention qui tombe du ciel, du flash inventif (Deforge, 1985).

Plusieurs approches transversales sont possibles : comprendre l'innovation c'est aussi faire appel, par exemple, à une notion assez complexe : l'imaginaire. Les discours, les récits, les fictions, les mythes (Perriault, 1989 ; Sfez, 2002) qui circulent autour de la technique véhiculent un imaginaire propice à l'innovation, sa conception, ses représentations, ses

\footnotetext{
${ }^{5}$ Pensons par exemple à la sociologie de la traduction développée par Michel Callon, Bruno Latour et Madeleine Akrich.
} 
usages. En retraçant l'histoire d'Internet, Patrice Flichy (2001) a bien montré l'importance du rôle de l'imaginaire à travers les utopies et idéologies qui ont circulé autour de cette innovation. Cet imaginaire organisé autour de l'idée d'un possible «village planétaire » est intéressant à prendre en compte pour comprendre l'univers dans lequel les concepteurs ont baigné, mais aussi pour analyser les discours qui ont accompagné les premiers temps d'Internet.

Autre exemple de transversalité pour terminer: celle qui consiste à montrer aux futurs ingénieurs l'importance que peuvent avoir les usagers, dans le processus d'innovation. Ce point de vue socio-économique (Von Hippel, 2005 ; Cardon, 2006) vient bousculer l'image d'une innovation qui se fait uniquement par les ingénieurs du service de R\&D puisqu'elle démontre que dans certains cas, les usagers ont eux aussi les compétences pour inventer, améliorer, expérimenter voire commercialiser des objets innovants. Ces usagers qu'Eric Von Hippel (2005) appelle « lead users » sont par exemple à l'origine de $80 \%$ des instruments scientifiques.

\section{Conclusion}

Les quelques pistes mentionnées ne sont certainement pas exhaustives. Elles impliquent en tout cas de repenser la formation et les approches pédagogiques. Comment passer du vœu pieu à la pratique ? Comment mettre en œuvre ces formations qui mobilisent de la part des enseignants des savoirs divers et qui les obligent à sortir des cadrages disciplinaires ? Comment en conséquence imaginer des modules de formation qui mobilisent des savoirs et savoir-faire si variés?

Un certain nombre d'obstacles subsistent. Le premier est d'ordre «culturel » : Jean Capelle disait dans les «Entretiens sur la technologie » en 1966 en citant Leonard de Vinci qu'il est " beaucoup plus facile de mépriser la technique que de se donner la peine de la comprendre » (Capelle, 1966 : 29).

Un autre frein tient au découpage disciplinaire de l'université française : comment valoriser un enseignement et une recherche (car les deux sont liés) qui ne cadrent pas avec les sections définies par le Conseil National des Universités et qui de fait placent les enseignantschercheurs dans des entre-deux préjudiciables à leur carrière? Aujourd'hui, il apparaît certainement plus facile de considérer l'innovation (et la technique en général) comme une boîte noire mais il nous faudra et ce, dans un court terme, bousculer ce type de croyances et proposer d'autres formations en lien avec les ambitions que nous voulons donner à nos ingénieurs.

\section{Bibliographie}

D'Alembert, (Jean), « Discours préliminaire », in Diderot et D'Alembert, L'Encyclopédie, pp. 19-120, Le Monde/Flammarion, Paris, 2010.

Berger, (Gaston), «Humanisme et technique », Revue de l'enseignement supérieur, $\mathrm{n}^{\circ} 1$, pp.5-10, janvier-mars 1958.

Capelle, (Jean), «Entretien » Dix entretiens sur la technologie, Ministère de l'Education Nationale, pp. 27-31, 1966.

Cardon, (Dominique), L'innovation par l'usage, 12 p., 2006. [Consulté le 26 avril 2011 sur http://vecam.org/article588.html]

Chabot, (Pascal), La Philosophie de Simondon, Vrin, Paris, 2003.

Chouteau, (Marianne), Forest, (Joëlle), Nguyen (Céline), «Il était une fois l'INSA... Un réseau d'hommes réformateurs », Actes du congrès de la SFHST, Editions Hermann, Paris, à paraître.

Deforge, (Yves), Technologie et génétique de l'objet industriel, Maloine, Paris, 1985. 
Faucheux (Michel), Forest (Joelle), « Stimulating a creative rationality to stimulate innovation », Creativity and Innovation Management, à paraître, 2011.

Felder, (Richard), Sheppard (Sheri), Smith (Karl), «A new journal for a field in transition », Journal of Engineering Education, vol 94, ${ }^{\circ}{ }^{1}$, pp. 7-10, january, 2005.

Flichy (Patrice), « La place de l'imaginaire dans l'action technique », Réseaux, n ${ }^{\circ}$ 109, pp.5273.

Grelon, (André), «Profil d'ingénieurs français 1950-1980», Le mouvement social, n¹63, avril-juin, p. 85, 1993.

JEE (Journal of Engineering Education), Special Report: The Research Agenda for the New Discipline of Engineering Education, Vol. 95, no. 4, pp.259-261, October 2006.

Lamard, (Pierre), Lequin, (Yves-Claude), La Technologie entre à l'Université : Compiègne, Troyes, Belfort-Montbéliard, Pôle éditorial de l’UTBM, Sévenans, 2006.

Latour, (Bruno), «Portrait de Gaston Lagaffe en philosophe des techniques », in Petites leçons de sociologie des sciences, pp. 14-24, La Découverte, Paris, 2006.

Lemaître, (Denis), «La recherche en SHS dans les écoles d'ingénieurs : une sécularisation des humanités classiques ? », in Faucheux M. et Forest J. (coord.) Les recherches en sciences humaines et sociales dans les écoles d'ingénieurs, pp. 63-73, Petra, Paris, 2007.

Longchambon, (Henri), "La formation des ingénieurs dans l'Université », Revue de l'enseignement supérieur, 1, janvier-mars, pp. 11-16, 1957.

National Research Council, Improving Engineering Design: Designing for Competitive Advantage, National Academy Press, Washington, 1991.

Perriault, (Jacques), La logique de l'usage: essai sur les machines à communiquer, L'Harmattan, Paris, 1989.

Sfez, (Lucien), Technique et idéologie, Seuil, Paris, 2002.

Shinn, (Terry), «Des corps de l'Etat au secteur industriel: genèse de la profession d'ingénieur, 1750-1920 », Revue Française de sociologie, XIX, pp. 39-71, 1978.

Sigaut, (Francois), «Haudricourt et la technologie », in Sigaut, F. (Dir.), La Technologie, science humaine, pp.9-34, Éditions de la Maison des Sciences de l'Homme, Paris, 1986.

Simon, (Herbert A), The Sciences of the Artificial, MIT Press, Cambridge, 1969.

Simondon, (Georges), Du mode d'existence des objets techniques, Aubier, Paris, 2001.

Sonntag, (Michel) and al., "Les questions de formation dans les Ecoles d'Ingénieurs. Une place pour la recherche ?», Recherches et Educations, n ${ }^{\circ} 1,2$ semestre 2008, pp. 121-144, [consulté sur http://rechercheseducations.revues.org, le 26 avril 2011].

Stiegler, (Bernard), Philosopher par accident, Galilée, Paris, 2004.

Vinck, (Dominique), «La médiation sociologique: entre éthique et pratique, la connaissance » in B Feltz, P. Goujon, B. Hériard Dubreuil, S. Lavelle et W Lesch (Ed) Ethique, technique et démocratie, pp. 253-270, 2007 [consulté le 26 avril 2011 sur: http://hal.archives-ouvertes.fr].

Von Hippel, (Eric), Democratizing innovation, MIT Press, Cambridge/London, 2005.

Waina, (Richard), « System design of curriculum », Engineering Education, October, pp. 97 100, 1969. 